\title{
Electroacupuncture at the Four Abdominal and Sacral Acupoints for Urinary Retention after Spinal Cord Injury: Study Protocol for a Randomized Controlled Trial
}

\section{Tong Liu}

Guangdong Second Hospital of TCM https://orcid.org/0000-0002-5185-2188

Jiani Yu

Guangdong provincial hospital of TCM

Weichuan Kuang

Guangzhou university of TCM

Xiaoyin Wang

Guangzhou university of TCM

\section{Xi Wen}

Guangzhou university of TCM

\section{Ye Jiang}

Guangzhou university of TCM

\section{Xiaojia Qiu}

Guangzhou university of TCM

\section{Yao Zeng}

Guangzhou university of TCM

\section{Guitao Zhang}

Guangzhou university of TCM

Yue Liu ( $\square$ liuyue2288@163.com )

Guangzhou university of TCM

\section{Study protocol}

Keywords: Electro-acupuncture, Four Abdominal and Sacral Acupoints, Urinary Retention, Spinal Cord Injury

Posted Date: July 24th, 2020

DOI: https://doi.org/10.21203/rs.3.rs-18358/v1 
License: (c) (i) This work is licensed under a Creative Commons Attribution 4.0 International License. Read Full License 


\section{Abstract}

Background $囚$ Urinary retention is a general and serious refractory complication after spinal cord injury (SCl). However, effective therapeutic approaches remain limited. Previous clinical and animal research have clarified that acupuncture could be beneficial to the recovery of bladder function, however, most of the previous trials are of low quality and high-quality studies are still necessary.

Methods: Here, we conduct a study design and protocol of a randomized, blinded, controlled study to evaluate the efficacy and safety of electroacupuncture at the "Four Abdominal and Sacral Acupoints" for urinary retention after $\mathrm{SCl}$. A total of 90 patients with residual urine above $300 \mathrm{ml}$ will be recruited in the trial and randomized into treatment group or control group. Patients in the treatment group will receive Electroacupuncture at the "Four Abdominal and Sacral Acupoints" combined with conventional treatment while patients in the control group will receive conventional treatment alone for 4 weeks. The primary outcomes are changes of residual urine volume at baseline, after the final treatment and 4 weeks after the final treatment. The secondary outcome measures will be the proportions of participants with residual urine decline $\geq 50 \%$ and efficacy rate after 4 weeks' treatment, as well as the proportion of patients with catheterization, Short-Form Health Survey Questionnaire (SF-36) and the incidence of symptomatic urinary tract infection at baseline, after the final treatment and 4 weeks after the final treatment. The safety will also be assessed by monitoring the incidence of adverse events and changes in vital signs during the study.

Discussion $\bigotimes$ Results from this trial will significantly support the application of electroacupuncture in the recovery of bladder function after $\mathrm{SCl}$. If found to be effective and safe, electroacupuncture will be a valuable complementary option for patients with urinary retention after $\mathrm{SCl}$.

Trial registration: Chinese Clinical Trial Registry: ChiCTR 1900022089. Registered on 24 March 2019, http://www.chictr.org.cn/showproj.aspx?proj=37161.

\section{Background}

Spinal Cord Injury (SCl) occupied a high morbidity and lethality all around the world. In China, it was reported that the prevalence of SCl was 37 per million, and multiple complications could appear after $\mathrm{SCl}$ [1]. Urinary retention is a common bladder dysfunction accompanied by $\mathrm{SCl}$, which was characterized by incomplete emptying after the patient has passed urine. About $74-80 \%$ of patients with $\mathrm{SCl}$ were reported suffering some degree of urinary retention within 1 year of injury [2-3]. Long-lasting urinary retention may induce physical and mental burden in patients, and worsely, it will cause significant complications, including recurrent urinary tract infections (UTIs) and progressive renal failure because of high pressures within the bladder [4]. Thus, it is an intractable issue in patients with $\mathrm{SCl}$ and effective therapies are in emergency need.

Although majority of therapies have been applied to treat urinary retention, including medication [5-6], bladder catheterization [7], physiotherapy [8]. However, all these approaches exhibited some 
disadvantages or side effects [9-10]. As a complementary and alternative therapy, acupuncture is an important component of traditional Chinese medicine and has been widely used in various diseases during the past thousands of years in China. Actually, acupuncture has been investigated in patients with urinary retention [11-13], however, most of these researches are of low quality and lack of sufficient sample size.

Thus, we present a study design and protocol of a randomized, blinded, controlled study to assess the efficacy and safety of electroacupuncture for urinary retention in patients with $\mathrm{SCl}$. The objective of this study was to determine if eletroacupuncture combined with conventional treatment is more effective than conventional treatment alone. The results of this study are expected to establish an optimal electroacupuncture procedure for urinary retention in patients with $\mathrm{SCl}$.

\section{Methods}

\section{Objective}

In this study, the efficacy of combined application of Electroacupuncture at the "Four Abdominal and Sacral Acupoints" and conventional treatment versus conventional treatment will be evaluated.

\section{Trial design and setting}

This study is a single-center, randomized controlled, assessor-blinded clinical trial which was devised following the Consolidated Standards of Reporting Trials (CONSORT) Statement recommendations [14]. The total study period for this trial is 8 weeks (Figure. 1), including 4-week treatment phase and 4-week follow-up phase.

It will be performed in GuangDong Second Hospital of Traditional Chinese Medicine. 90 patients who meet the eligibility criteria and sign an informed consent form will be randomly devided into two groups to receive either electroacupuncture at the Four abdominal and sacral acupoints plus conventional treatment or conventional treatment alone in a 1:1 ratio. Electroacupuncture will be performed 30 min per day, 5 days per week for 4 weeks. Doctors with more than 5 years of clinical experience will be allowed to perform the interventions. After 4 weeks, Outcome measurements including residual urine volume proportions of participants with residual urine decline $\geq 50 \%$ efficacy rate\proportion of patients with catheterization $\square$ Short-Form Health Survey Questionnaire (SF-36) and the incidence of recurrent symptomatic urinary tract infection will be analysed by doctors who was blind to the allocation of the patients. The flowchart of the trial is shown in Figure. 2.

\section{Participants}

\section{Recruitment strategies}

Inpatients at acupuncture departments in GuangDong Second Hospital of Traditional Chinese Medicine will be recruited mainly in this RCT. Additionally, posters will be put in the hospitals and a Chinese 
multipurpose social media named WeChat will be applied to recruit. Brief descriptions of eligible criteria, the free acupuncture treatments and the possible risks of the trial will be marked and all patients have the right to participate or drop out at any time, and will be required to sign the informed consent before the trial begin.

Eligibility criteria: inclusion criteria

The inclusion criteria will be as follows.

1.Meet the diagnostic criteria for Urinary Retention after SCl;

2.Aged 18-60 years old, male or female;

3. more than $300 \mathrm{ml}$ residual urine were detected in their bladder;

4.patients had clear consciousness and difficulty in urination;

5.patients complained of distention and pain in lower abdomen;

6.signed informed consent.

The exclusion criteria will be as follows.

1.Urinary retention not induced by SCl;

2.Clinical course more than 1 year;

3.Combination of heart, liver or kidney failure endanger the safety of life at any time;

4.Younger than 18 years or older than 60 years;

5.Those who do not receive electroacupuncture therapy;

6.Women with pregnancy and lactation.

Randomization and allocation

Random numbers will be generated by the random number generator in the SPSS statistical software package (Version 22.0, SAS Institute Inc.). Another specified researcher who is not involved in the study was responsible for it.

The allocation of participants will be sealed in a sequentially numbered opaque envelope. If the participant meets the inclusion criteria and signs informed consent, the above specified researcher will sequentially give the sealed random number envelope to the physician, who will open the envelope and allocate the participant to either electroacupuncture at the Four abdominal and sacral acupoints plus conventional treatment group or conventional treatment alone group according to the random number. 
Blinding

Because of the add-on study design, a single-blinded method will be used. While the participants and practitioners cannot be blinded, we blinded the outcome assessors, data manager, and statistics analyzer. The assessor will be instructed not to communicate with participants about the possibility of their treatment. James et al.'s blinding index will be evaluated after the completion of the study to evaluate the success of blinding [15].

Intervention

Study schedule

Treatment group intervention

Patients in the treatment group will receive both electroacupuncture combined with conventional treatment. Electroacupuncture manipulation was carried out in two steps: First, patients will be asked to take a supine position. Following disinfection of skin with 75\% alcohol, bilateral "Shuidao" (ST28) acupoints $\square$ Guanyuan (RN4) acupoint and Zhongji (RN3) acupoint (Four abdominal acupoints) will be stimulated using disposable and stainless needles $(75 \mathrm{~mm}$ long and $0.30 \mathrm{~mm}$ in diameter, Suzhou Huanqiu Acupuncture Medical Appliance, Suzhou, China), inserted to a depth of 40-55mm until needling sensation transmit towards perineum and bladder. Then needles in the acupoints of bilateral ST29 as well as the needles in the acupoints of RN3 and RN4 will be connected to an electrical stimulator (SDZ-V EA; Huatuo, Suzhou, China) with a frequency of $2 / 10 \mathrm{~Hz}$ and current of $1 \mathrm{~mA}$ for 15 min, respectively. Secondly, the patients were placed in prone position, four sacral points were selected with the two upper points are located by the two edges of the sacrum on a level with the fourth sacral foramina while the locations of the two lower points are about $1 \mathrm{~cm}$ bilateral to the tip of the coccyx (Fig. 3, cited from Wang et al). The two upper points were inserted perpendicularly to a depth of 60 to $70 \mathrm{~mm}$ to produce a sensation referred to the urethra or the anus by stimulating the main trunk of the PN with disposable and stainless needles (75 mm long and $0.30 \mathrm{~mm}$ in diameter, Suzhou Huanqiu Acupuncture Medical Appliance, Suzhou, China). The lower points were inserted obliquely toward the ischiorectal fossa to a depth of 60 to $70 \mathrm{~mm}$ to produce a sensation referred to the urethra by stimulating the perineal nerve with acupuncture needles of the same model. Then needles in the acupoints of bilateral two upper points as well as the needles in the acupoints of bilateral two lower points will be connected to an electrical stimulator (SDZ-V EA; Huatuo, Suzhou, China) with a frequency of 2/10 Hz and current of $1 \mathrm{~mA}$ for 15 min, respectively. Electroacupuncture was manipulated once daily, 5 times a week and lasted for 4 weeks.

Conventional treatment refers to clean intermittent catheterization (CIC), which was carried out as follows: First, clean the penis/vulva of patients and open the urethra, then lubricate the catheter and insert the catheter gently. Stop advancing the catheter until urine flow starts and remove the catheter until urine flow stops. CIC was carried 6-8 times per hour, which based on fluid intake and bladder capacity of patients. CIC coud be ceased if bladder residual urine volume is less than $100 \mathrm{ml}$. 
Control group intervention

Patients in control group will receive $\mathrm{CIC}$ treatment following the same protocol used in the treatment group. Electroacupuncture will not be administered.

Discontinuing interventions

The trial will be ceased if one of the following conditions appeared: (1) a serious poststroke complication arises; or (2) recurrent stroke or any other severe condition occurs leaving the patient in a critical condition.

\section{Outcome measures $\rrbracket$}

The primary outcomes are changes of residual urine volume at baselinelafter the final treatment and 4 weeks after the final treatment. The secondary outcome measures will be the proportions of participants with residual urine decline $\geq 50 \%$ and efficacy rate after 4 weeks'treatment, as well as the the proportion of patients with catheterization $\square$ Short-Form Health Survey Questionnaire (SF-36) and the incidence of recurrent symptomatic urinary tract infection at baselinelafter the final treatment and 4 weeks after the final treatment.

Any adverse events, including acupoint hematoma, infection, dizziness and apostasies will be evaluated during the whole treatment by the researcher. If any severe adverse events occurring, acupuncture intervention will be ceased immediately and the principal investigator will be informed to take proper actions. 1 month will be followed up after the trial in case of subsequent adverse events.

\section{Safety assessment}

Any adverse events, including acupoint hematoma, infection, dizziness and apostasies will be evaluated during the whole treatment by the researcher. If any severe adverse events occurring, acupuncture intervention will be ceased immediately and the principal investigator will be informed to take proper actions. 1 month will be followed up after the trial in case of subsequent adverse events.

Sample size calculation

The trial is designed to determine the role of electroacupuncture at the Four abdominal and sacral acupoints for urinary retention in patients with $\mathrm{SCl}$ and prove that electroacupuncture at the Four abdominal and sacral acupoints combined with conventional treatment is superior to conventional treatment alone. Therefore, residual urine volume change will be used as an evaluation index. According to our previous pilot study, the change of residual urine volume before and after treatment in the treatment group was shown to be 194.00 $16.91(n=5)$ and that in control group was $184.50 \pm 14.35(n=$ 5). Sample size was estimated using the following formula: 
$n=\left[\frac{\left(Z_{\alpha / 2}+Z_{\beta}\right) \sigma}{\delta}\right]^{2}\left(\frac{1}{Q_{1}}+\frac{1}{Q_{2}}\right)$

where $n$ represents the number of samples required, $n=n_{1}+n_{2}, Q_{1}=n_{1} / n, Q_{2}=n_{2} / n$ with a significance level $(a=0.05)$ of a two-sided two-sample t-test and $80 \%$ power to detect a difference between the two groups. Thus, a total sample size of 90 patients will be recruited allowing for $10 \%$ of attrition, with 45 in each.

Statistical analysis

SPSS 20.0 (IBM SPSS Statistics, IBM Corp, Somers, New York, USA) will be used to analyze the data. Quantitative data will be presented as mean \pm SD. GCS $\square$ Barthel and FuglMeyer scores will be conducted between the two groups by a superiority independent sample t-test with a $95 \% \mathrm{Cl}$. The incidence of complications and adverse events will be compared by $\chi 2$ test. Any missing data will be replaced by the last measured value. For all analyses, $P$ values of less than 0.05 will be considered statistically significant.

Quality control

Before the trial, all the acupuncturists $\llbracket$ nurses and assessors will be trained strictly in order to guarantee homogeneity in the measurement data and ensure high-quality data results. The training content will include study protocol, diagnosis, inclusion and exclusion criteria, recording method of CRF, the location of the acupoints, acupuncture operation techniques, disposal of bleeding. All the study data will be recorded on the case report form. Dropouts and withdrawals from the study will be recorded in detail based on the intervention and follow-up periods. Data will be uploaded and verified by other two researchers who were not involved in the trial. This trial will be monitored by the Scientific Research Department of GuangDong Second Hospital of Traditional Chinese Medicine every one week.

\section{Discussion}

Discordance between the function of detrusor and urethral sphincter lead to urinary retention after SCl. Long-term of urinary retention could induce renal impairments and upper urinary tract infections [16-17]. Here, we conducted a study to compare the effect of eletroacupuncture combined with conventional treatment with conventional treatment alone to determine if eletroacupuncture could improve the function of spinal cord. In our trial, strict quality control procedures have been applied to avoid bias, such as randomization and allocation concealment, assessor blinding and adequate sample size. However, methodological limitations still exist. Due to the nature of acupuncture manipulation, the therapists and the participants cannot be blinded in this study.

In this study, 8 acupoints including 4 abdominal and 4 sacral acupoints were selected. Shuidao (ST28) Guanyuan (RN4) and Zhongji (RN3) acupoints are widely used in treating urinary retention according to tradiontional meridian theory. Guanyuan (RN4) and Zhongji (RN3) acupoints belongs to Ren meridian. 
Guanyuan (RN4) is the "Mu point" of small intestine while Zhongji (RN3) is the "Mu point" of bladder. Deep insertion of the above four acupoints could stimulate pelvic plexus and induce a needling sensation transmit towards perineum and bladder. Studier indicated that acupuncture at Shuidao (ST28) and Guanyuan (RN4) acupoints could enhance activity and excitability of detrusor by repairing damaged central nervous cells and neural pathways [18-19]. The 4 sacral acupoints was proposed by a doctor named Wang Siyou [20] who fond the site about $1 \mathrm{~cm}$ bilateral to the sacrococcygeal joint is close to the main pudendal nerve trunk, while in the ischiorectal fossa, the PN branches into 1) the perineal nerve innervating the PFM and the skin of the labium majus, and 2) the dorsal nerve of the clitoris, which innervates the skin of the clitoral shaft [21]. Therefore, the sensation is only referred to the urethra when a long needle is inserted beside the tip of the coccyx laterally toward the ischiorectal fossa to make the needle tip reach the perineal nerve.

In conclusion, this study will provide solid evidence of the role of combined application of electroacupuncture in improving urinary retention after $\mathrm{SCl}$.

\section{Abbreviations}

SCl: spinal cord injury; SF36: Short-Form Health Survey Questionnaire; CIC: clean intermittent catheterization; CONSORT: Consolidated standards of reporting trials; CRF: Case report form

\section{Declarations}

Trial status

This is protocol version1.0, version date is 2019.3.1. Participants are being recruited. Recruitment began on 1 July 2019. The trial is planned to be completed by 31 December 2021, 1 year later than the actual information we registered.

Acknowledgments

We are thankful to Prof. Nie Bin, GuangDong Second Hospital of Traditional Chinese Medicine, for scientific designment.

\section{Funding}

This report is financially supported by the National Science Foundation for Young Scientists of China, No. 81704179. The funder have no role in study design, data collection and analysis, decision to publish, or preparation of the manuscript.

Availability of data and materials

The results of this trial will be presented through scientific reports or journals. 


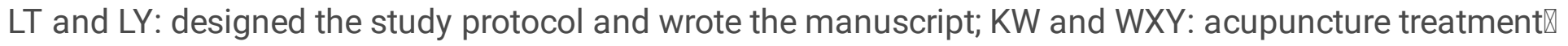
WX and JY: outcome assessment; YJ: statistical analysis; QXJ: ethical approval; ZY and ZGT: follow-up. All authors approve the final version of the paper. The corresponding author has the sole responsibility of submission of the manuscript for publication.

Ethics approval and consent to participate

We strictly follow the principles of the medical ethics of the Declaration of Helsinki with the approval of the Ethics and Research Committee of GuangDong Second Hospital of Traditional Chinese Medicine, China.llf there are any protocol modifications, we will report to the Ethics and Research Committee for approval. All patients will be recruited from the Department of Acupuncture and Rehabilitation, GuangDong Second Hospital of Traditional Chinese Medicine. Informed consent will be obtained from all study participants and their legal guardians. Participant information will be protected. All experimental data will be stored in a secure storage area with access limited to the researchers alone.

Consent for publication: Not applicable.

Competing interests: The authors declare that they have no competing interests.

Provenance and peer review: Not commissioned; peer reviewed for ethics and funding approval prior to submission.

\section{References}

[1] Xingyue C, Dong C, Chunhui C, et al. The epidemiology and disease burden of traumatic spinal cord injury in China: a systematic review[J]. J Evid Based Med, 2018, 18(02):143-150.

[2] Ginsberg D. The epidemiology and pathophysiology of neurogenic bladder. Am J Manag Care. 2013, 19(10 Suppl): s191-196. $\otimes$

[3] Manack A, Motsko SP, Haag-Molkenteller C, et al. Epidemiology and healthcare utilization of

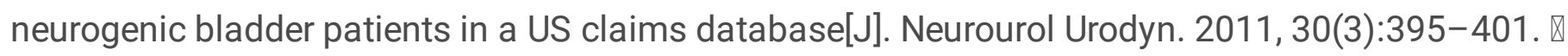

[4] Gormley EA. Urologic complications of the neurogenic bladder[J]. Urol Clin North Am. 2010, 37(4):601-607. 区

[5] Sharifi SH, Mokarrar MH, Khaledi F, et al. Does sildenafil enhance the effect of tamsulosin in relieving acute urinary retention? [J] Int Braz J Urol. 2014, 40:373-378.

[6] Elbendary M, El-Gamal OM, Soliman MG, et al. Role of combined use of ketoconazole and tamsulosin in management of acute urinary retention due to benign prostatic obstruction (a randomized controlled trial)[J]. Prostate Cancer Prostatic Dis. 2013, 16:362-366. 
[7] Brouwer TA, Rosier PF, Moons KG, et al. Postoperative bladder catheterization based on individual bladder capacity: A randomized trial[J]. Anesthesiology. 2015, 122:46-54.

[8] Moossdorff-Steinhauser HF, Berghmans B. Effects of percutaneous tibial nerve stimulation on adult patients with overactive bladder syndrome: a systematic review[J]. Neurourol Urodyn. 2013, 32:206-214.

[9] Rahman MA, Alam MM, Shahjamal S, et al. Predictive value of urine cultures in evaluation of bacterial colonization of ureteral stents[J]. Mymensingh Med J. 2012, 21:300-305.

[10] Lombardi G, Del Popolo G. Clinical outcome of sacral neuromodulation in incomplete spinal cord injured patients suffering from neurogenic lower urinary tract symptoms[J]. Spinal Cord. 2009, 47:486491.

[11] Can Z, Ren-Fu Q, Le C, et al. Effects on urinary retention after spinal cord injury treated with acupuncture at Zhibian (BL54)and Shuidao (ST28) with elongated needle[J]. Zhongguo Zhen Jiu, 2019, 39(4):359-363.

[12] Xu X, Xu Y. Clinical efficacy on neurogenic bladder after spinal cord injury treated with rehabilitation training and acupuncture-moxibustion[J]. Zhongguo Zhen Jiu, 2015, 35(7):670-673.

[13] Jiang Q, Yajie Z, Xiuxiu S, et al. Effects of acupuncture intervention at different stages on urinary function reconstruction of neurogenic bladder after spinal cord injury[J]. Zhongguo Zhen Jiu, 2015, 35(2):132-136.

[14] Schulz KF, Altman DG, Moher D. CONSORT 2010 statement: updated guidelines for reporting parallel group randomized trials[J]. BMC Med. 2010, 8:18.

[15] James KE, Bloch DA, Lee KK, et al. An index for assessing blindness in a multi-centre clinical trial: disulfiram for alcohol cessation-a VA cooperative study[J]. Stat Med. 1996, 15:1421-1434.

[16] Sievert KD, Amend B, Gakis G, et al. Early sacral neuromodulation prevents urinary incontinence after complete spinal cord injury[J]. Ann Neurol. 2010, 67(1):74-84.

[17] Schneider MP, Hughes FM Jr, Engmann AK, et al. A novel urodynamic model for lower urinary tract assessment in awake rats[J]. BJU Int. 2015, 115(suppl 6): 8-15.

[18] Feng Z, Yi F, Rui Z, et al. Observation on the Central Afferent Pathway of "Guanyuan"(RN4) under Normal and Pathological States and the Influence of Electroacupuncture[J]. Acupuncture Research, 2008 (03):147-153.

[19] Wang JH, Chen BG, Yin J, et al. Effect of Electroacupuncture of Different Acupoints on the Excitability of Detrusor Muscle and the Expression of BDNF and TrkB in the Spinal Cord of Rats with Urinary Retention due to Spinal Cord Injury[J]. Acupuncture Research, 2009, 34(6):387-392. 
[20] Wang SY, Zhang SJ. Simultaneous perineal ultrasound and vaginal pressure measurement prove the action of electrical pudendal nerve stimulation in treating female stress incontinence[J]. BJU Int. 2012,110(9):1338-1343.

[21] Wang SY, Lu J, Feng X, et al. Efficacy of Electrical Pudendal Nerve Stimulation versus Transvaginal Electrical Stimulation in Treating Female Idiopathic Urgency Urinary Incontinence[J]. J Urol. 2017, 197(6):1496-1501.

\section{Tables}

Table1 Description of the applied acupuncture points

\begin{tabular}{ll}
\hline Acupoints & Descriptions \\
\hline RN4: Guanyuan & 3 cun below the belly button \\
ST28: Shuidao & 2 cun next to RN4 \\
& 4 cun below the belly button \\
RN3: Zhongji & located by the two edges of the sacrum \\
Two upper sacral acupoints & \\
Two lower sacral acupoints & about $1 \mathrm{~cm}$ bilateral to the tip of the coccyx \\
\hline
\end{tabular}

\section{Figures}




\begin{tabular}{|c|c|c|c|c|c|}
\hline & \multicolumn{5}{|c|}{ Study period } \\
\hline & Enrolment & Allocation & \multicolumn{3}{|c|}{ Postallocation } \\
\hline Time point* & $-\mathrm{t} 1$ & $\mathrm{t} 0$ & 1 & 4 & 8 \\
\hline \\
\hline \multirow{3}{*}{$\begin{array}{l}\text { Eligibility screen } \\
\text { Informed consent } \\
\text { Allocation }\end{array}$} & $x$ & & & & \\
\hline & $x$ & & & & \\
\hline & & $x$ & & & \\
\hline \multicolumn{6}{|l|}{ Interventions: } \\
\hline \multicolumn{6}{|l|}{ Electroacupuncture treatment } \\
\hline $\begin{array}{l}\text { Conventional treatment } \\
\text { group) }\end{array}$ & & & & & \\
\hline \multicolumn{6}{|l|}{ Assessment: } \\
\hline changes of residual urine volume & & $x$ & & $x$ & $x$ \\
\hline $\begin{array}{l}\text { the proportions of participants with } \\
\text { residual urine decline } \geq 50 \%\end{array}$ & & & & $x$ & \\
\hline efficacy rate & & & & $x$ & \\
\hline $\begin{array}{l}\text { the proportion of patients with } \\
\text { catheterization }\end{array}$ & & $x$ & & $x$ & $x$ \\
\hline SF-36 & & $x$ & & $x$ & $x$ \\
\hline $\begin{array}{l}\text { the incidence of symptomatic } \\
\text { urinary tract infection }\end{array}$ & & $x$ & & $x$ & $x$ \\
\hline
\end{tabular}

Figure 1

Schedule of enrollment, interventions, and assessments 


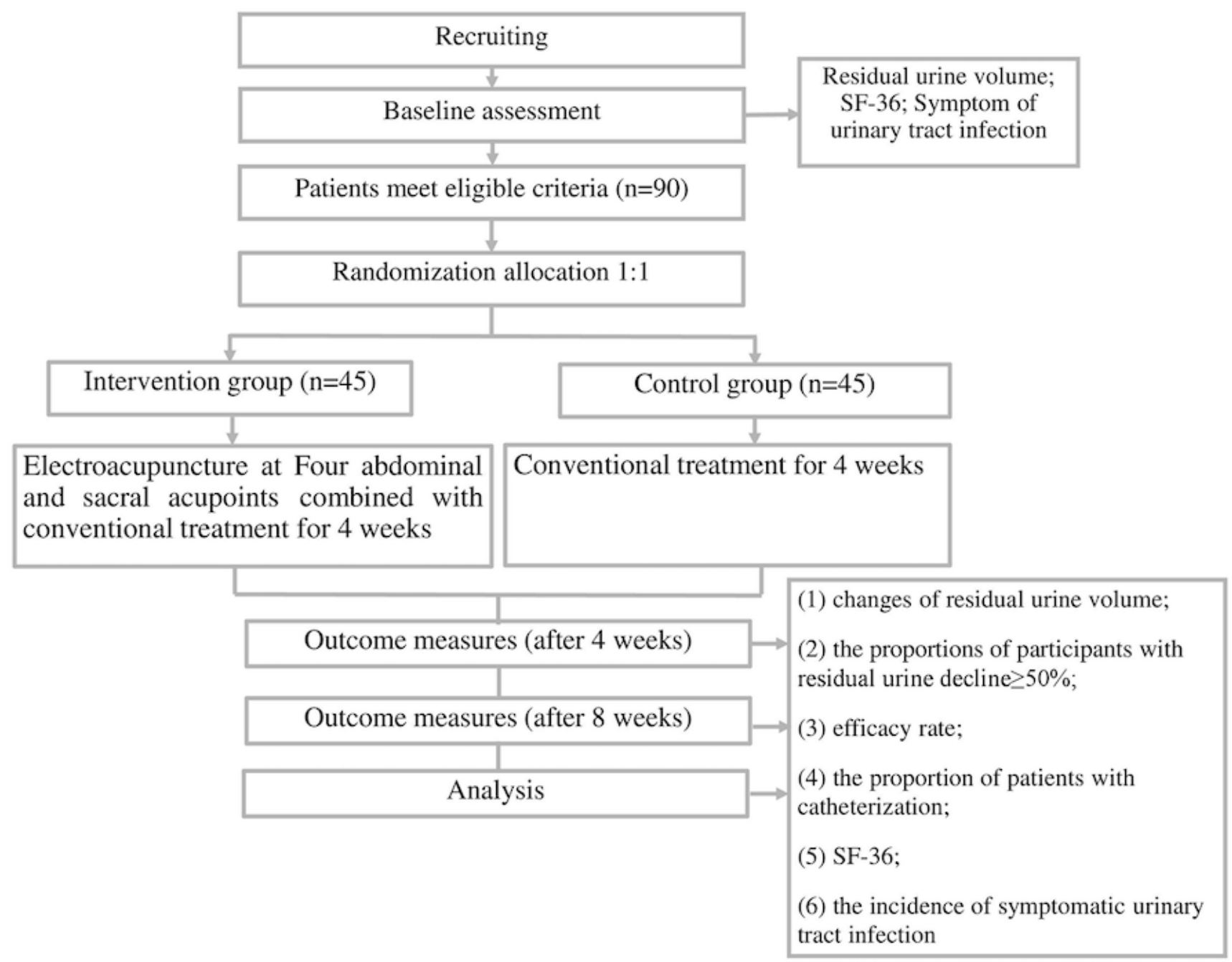

Figure 2

Flow chart of the study procedure 


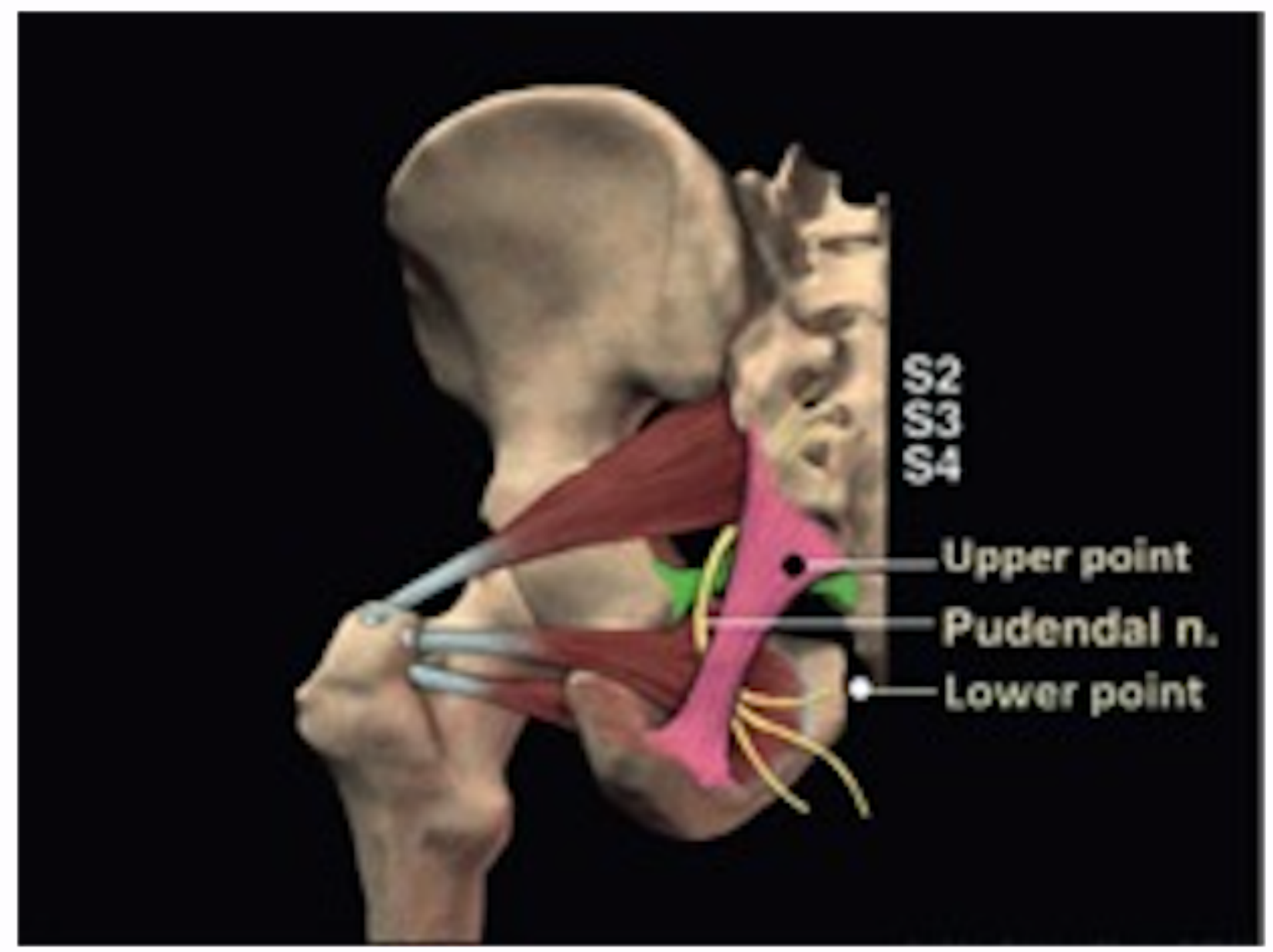

Figure 3

Four sacral acupoints. n.: nerve. (Cited from Wang et al)

\section{Supplementary Files}

This is a list of supplementary files associated with this preprint. Click to download.

- checklist.doc 